\title{
ULTRAMAP: THE ALL IN ONE PHOTOGRAMMETRIC SOLUTION
}

\author{
Alexander Wiechert, Michael Gruber, Konrad Karner \\ Vexcel Imaging Austria / Microsoft Photogrammetry \\ Anzengrubergasse 8/4, $8010 \mathrm{Graz} /$ Austria \\ \{alwieche, michgrub, kkarner\}@ microsoft.com
}

III/2: 3D Point Cloud Processing

KEY WORDS: Photogrammetry, Digital, Camera, Large Format, Mapping, Dense Matching

\begin{abstract}
This paper describes in detail the dense matcher developed since years by Vexcel Imaging in Graz for Microsoft's Bing Maps project. This dense matcher was exclusively developed for and used by Microsoft for the production of the 3D city models of Virtual Earth. It will now be made available to the public with the UltraMap software release mid-2012. That represents a revolutionary step in digital photogrammetry. The dense matcher generates digital surface models (DSM) and digital terrain models (DTM) automatically out of a set of overlapping UltraCam images. The models have an outstanding point density of several hundred points per square meter and sub-pixel accuracy and are generated automatically. The dense matcher consists of two steps. The first step rectifies overlapping image areas to speed up the dense image matching process. This rectification step ensures a very efficient processing and detects occluded areas by applying a back-matching step. In this dense image matching process a cost function consisting of a matching score as well as a smoothness term is minimized. In the second step the resulting range image patches are fused into a DSM by optimizing a global cost function. The whole process is optimized for multi-core CPUs and optionally uses GPUs if available. UltraMap 3.0 features also an additional step which is presented in this paper, a complete automated true-ortho and ortho workflow. For this, the UltraCam images are combined with the DSM or DTM in an automated rectification step and that results in high quality true-ortho or ortho images as a result of a highly automated workflow. The paper presents the new workflow and first results.
\end{abstract}

\section{Introduction}

Digital aerial cameras require specific software to process the raw images collected by the camera. Since its introduction in 2003, UltraCam has been shipped with a software package called OPC to perform the UltraCam specific image processing. A revolutionary step has been undertaken with the announcement of UltraMap Version1.0 in 2010, introducing new software for the image processing for the UltraCam camera series.

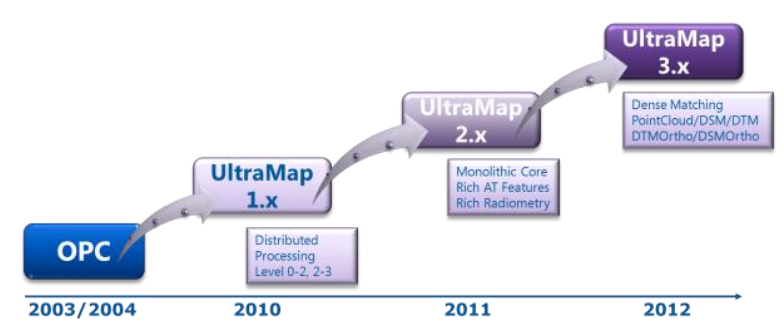

Figure 1. OPC/UltraMap Software Roadmap.

UltraMap supports all UltraCam cameras and extends the workflow into a full photogrammetric workflow by a seamless integration of aero triangulation and bundle adjustment functionality into the existing image processing workflow.

UltraMap is designed to handle all kind of projects ranging from projects with a few hundred images up to projects with ten thousands of images and it is optimized for UltraCam images. For this, it implements a new, revolutionary technology and concept of image handling, a direct spin off from the latest available Microsoft technology. Outstanding features such as automated distributed processing, automated tie point matching, monolithic stitching and project based color balancing have been implemented since its release. The figure below shows the basic modules and the basic workflow of the current UltraMap software. 


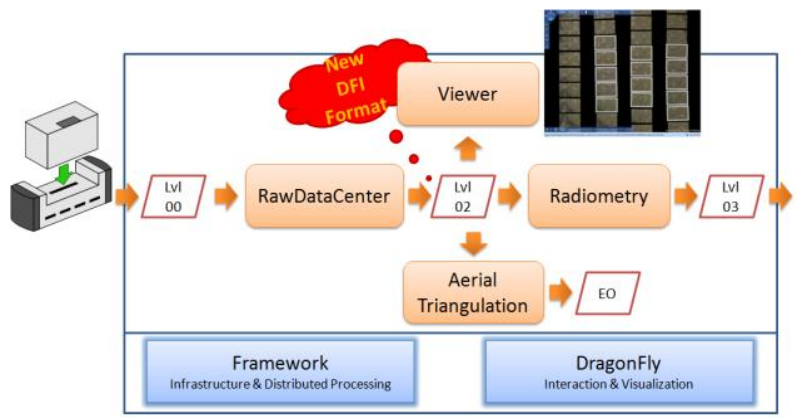

Figure 2. UltraMap concept and modules.

With the upcoming version of UltraMap 3.0 two new modules will be added to further extend the workflow. These two modules provide revolutionary new features, namely the automated generation of point clouds, digital surface models (DSM), digital terrain models (DTM), DSMOrtho images and DTM Ortho images, all derived from a set of overlapping UltraCam images. Results from the basic image processing and the aero triangulation are used by the new modules to generate a point cloud, then a DSM, then a DTM and then two different ortho images, the so called DSMOrtho (images rectified by a DSM) and DTMOrtho (images rectified by the DTM).

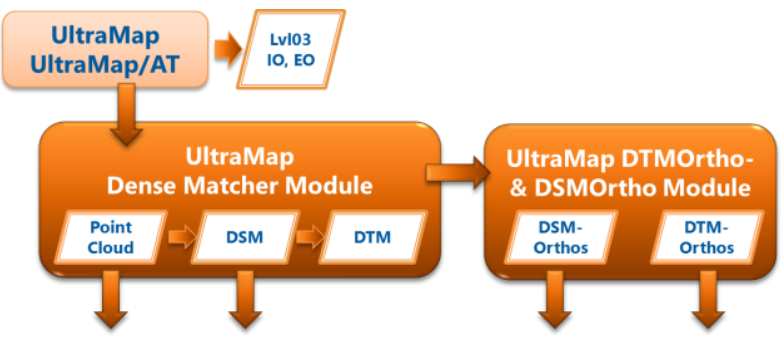

Figure 3. New UltraMap 3.0 modules

The processing is being kicked-off automatically after the aero triangulation and fully supports the automated distributed processing and the full 16-bit workflow. The new modules support processing on GPU(s) if available in the system. Visual output and QC are smoothly integrated into the existing viewer.

\section{Dense Matching and 3D Point Clouds}

A significant change in photogrammetry has been achieved by Multi-Ray Photogrammetry which became possible high performance digital aerial camera such as UltraCam and a fully digital workflow by software systems such as UltraMap. The cameras enable a significantly increased forward overlap as well as the ability to collect more images but literally without increasing acquisition costs. These highly redundant image datasets enable to generate new products such as point clouds highly automated and robust.
However, Multi-Ray Photogrammetry in a first step is not a new technology; it is a specific flight pattern with a very high forward overlap (80\%, even 90\%) and an increased sidelap (up to 60\%). The result is this highly redundant dataset that allows automated "dense matching" to generate high resolution, highly accurate point clouds from the imagery by matching the pixels of the overlapping images automatically.
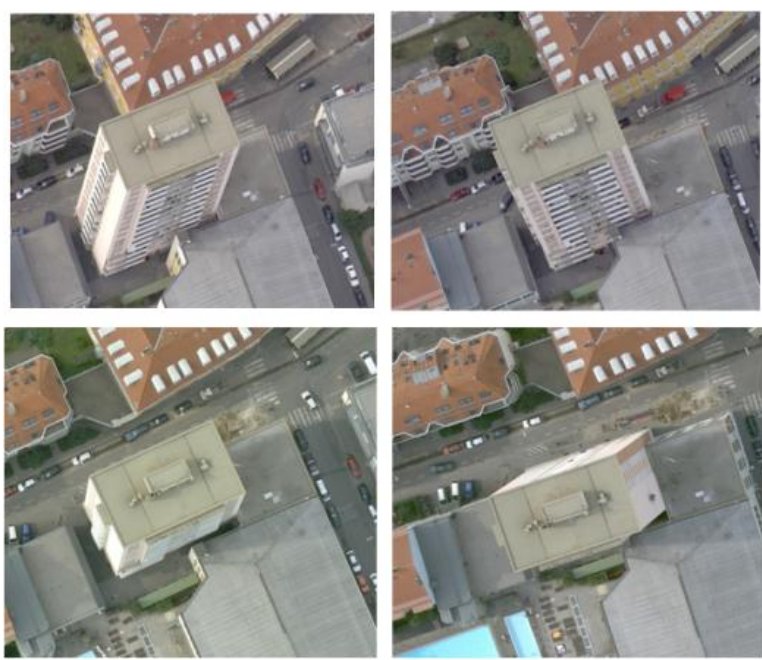

For the point cloud generation, the dense matcher analyzes the images and calculates a range image for each pixel on the ground from each stereo pair covering the pixel on the ground. The location $(\mathrm{x}, \mathrm{y}$,) of the pixel is defined by its position in the geo-referenced image; the range image defines a z-value for each pixel. Due to the highly redundant data set (thanks to the high forward and sidelap), usually multiple stereo pairs exist which cover one pixel on the ground. Thus multiple range images can be processed by the dense matcher who leads to multiple z-vales per pixel. That makes the whole process very robust and increases accuracy of the derived z-value.

The 3D point cloud generated by the dense matcher of UltraMap has a point density of several hundred points per square meter and thus is much denser than any airborne Lidar scanning point cloud. 


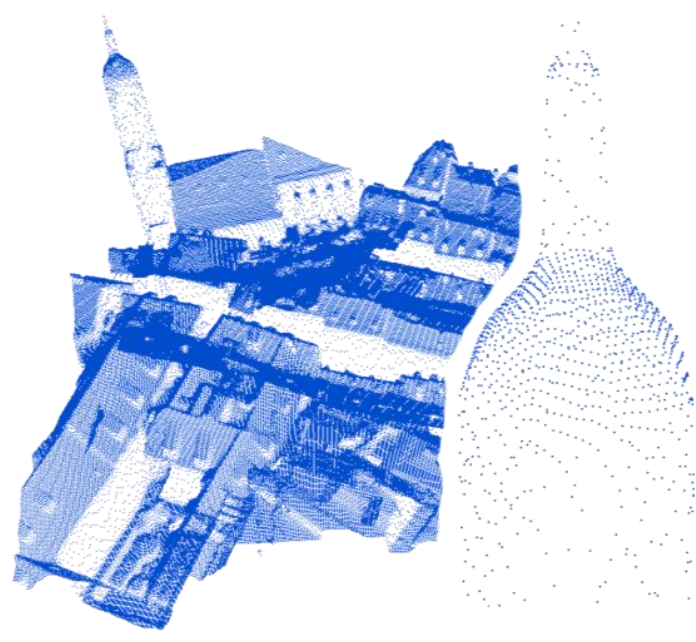

Figure 4. High resolution 3D point cloud generated by the dense matcher module of UltraMap

Figure 4 shows the point cloud generated by UltraMap based on a set of overlapping UltraCam images. The point density is around 300 points per square meter. For better visualization only a quarter of all points are shown.

The achievable height accuracy of the point cloud is better than the GSD, thus a $10 \mathrm{~cm}$ imagery leads to $<10 \mathrm{~cm}$ height accuracy of the resulting digital surface model. The quality of the automated dense matching process depends significantly on the camera and the structure of the terrain. Geometric stability and radiometric dynamic of the camera have a direct impact on the matching results.

\section{DSM and DTM}

Once the DSM has been processed out of the images, the DSM and the original image can be combined in a trueortho projection which results on a so called true-ortho image. This is an image with no perspective view as each pixel of the image has been (true-) ortho rectified by a surface model and not by a terrain model like the standard ortho image. True-ortho images have a significant benefit in some applications as they do not have any leaning objects.

Figure 5 shows the shaded relief of a DSM which has been generated from the point cloud. Remarkable is the high level of detail and also the very sharp building edges. The white spot at the lower left corner is the church tower shown in detail in figure 4 .

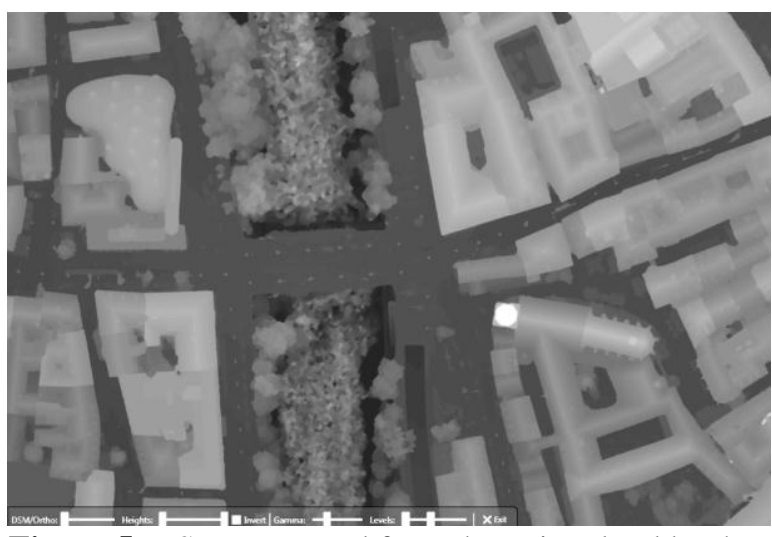

Figure 5. DSM generated from the point cloud by the UltraMap dense matcher module

The next step after the DSM generation is the processing of a DTM. The DTM will be processed out of the DSM using a "Winston-Salem" algorithm developed by Microsoft which utilizes computer vision based classification for the terrain filtering. Figure 6 shows the DSM (left) and the filtered DTM (right).
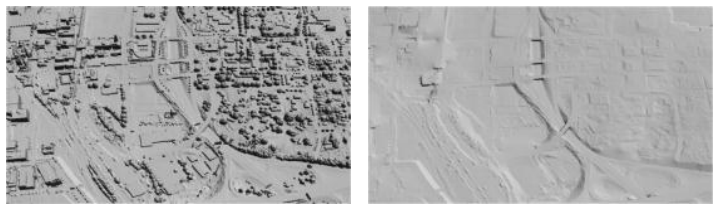

Figure 6. High-resolution DSM (left) and DTM (right) processed using automated dense matching with UltraCam images and UltraMap processing software

\section{DSMOrtho and DTMOrtho}

An additional new UltraMap 3.0 module then combines the image data and the DSM or DTM and generates automatically either a DSMOrtho or a DTMOrtho image including automated seamline generation. Figure 7 shows a DSMOrtho image, figure 8 a DTMOrtho image.

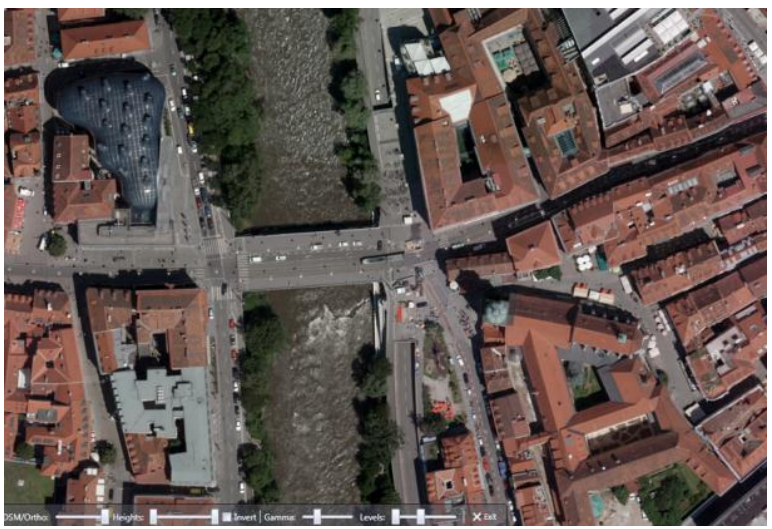

Figure 7. High resolution DSMOrtho, generated automatically by UltraMap 
Remarkable are the very sharp edges of the buildings and the high level of details of the roof structures with literally no artifacts of the DSMOrtho. The reason is the outstanding quality of the underlying DSM thanks to the high point density of the point cloud.

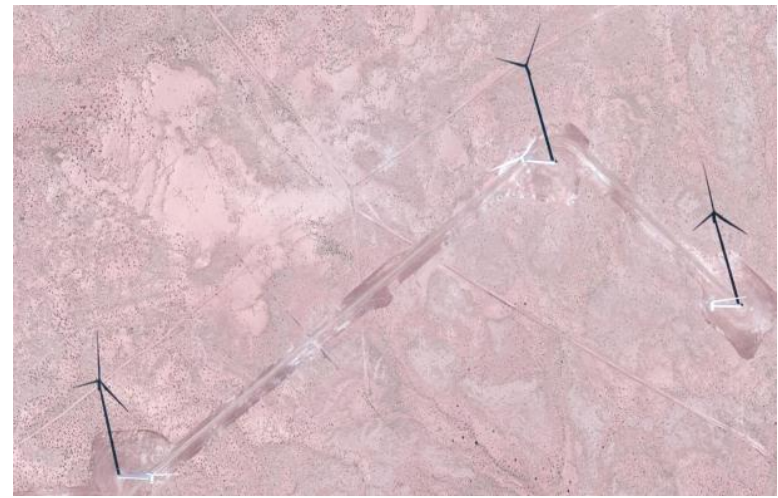

Figure 8. High resolution DTMOrtho, generated automatically by UltraMap

\section{References}

Wiechert et al, 2012: The power of multi-ray photogrammetry - UltraMap 3.0, Proceedings of the American Society for Photogrammetry \& Remote Sensing, 19-23 March 2012, Sacramento, CA.

Ladstaedter et al, 2010: Monolithic Stitching: One Sensor Geometry For Multiple Sensor Cameras, Proceedings of the American Society for Photogrammetry \& Remote Sensing, 26-30 April, 2010, San Diego, CA

Wiechert, A., Gruber M., Ponticelli M. (2011) UltraCam Eagle, the new Super-Large Format Digital Aerial Camera, Proceedings of the American Society for Photogrammetry \& Remote Sensing, 1-5 May, 2011, Milwaukee, WI.

Gruber, M. et al. 2012: UltraCam Eagle, understanding the new Sensor, Proceedings of the American Society for Photogrammetry \& Remote Sensing, 19-23 March 2012, Sacramento, CA. 